\title{
Congruency effects in the letter search task: Semantic activation in the absence of priming
}

\author{
Keith A. Hutchison and Frank A. Bosco \\ Montana State University, Bozeman, Montana
}

\begin{abstract}
Semantic priming is typically eliminated when participants perform a letter search on the prime, suggesting that semantic activation is conditional upon one's attentional goals. However, in such studies, semantic activation (or the lack thereof) is not measured during the letter search task itself but, instead, is inferred on the basis of the responses given to a later target. In the present study, direct online evidence for semantic activation was tested using words whose meaning should bias either a positive or a negative response (e.g., present vs. absent). In Experiment 1, a semantic congruency effect was obtained, with faster responses when the word meaning matched the required response. Experiment 2 replicated the congruency effect while, simultaneously, showing the elimination of semantic priming. It is concluded that letter search does not affect the initiation of semantic activation. Possible accounts for the elimination of priming following letter search include activation-based suppression and transfer-inappropriate processing.
\end{abstract}

Some mental processes require enormous effort to perform, and others we can do fairly easily; still others seem to happen whether we want them to or not. Suppose that while riding a subway, you see a billboard sign that reads "say no to drugs." Our usual tendency is just to read the words; however, if we had the desire (and/or had too much free time), we could perform other operations, such as generate a pig Latin version (e.g., aysay onay otay rugsday), generate rhyming words (e.g., lay low you thugs), or count the number of vowels in the sentence (i.e., four). Although reading the sentence seems unavoidable, these other operations require mental effort.

The idea that certain mental activities may be unavoidable has intrigued researchers since the 1970s (Hasher \& Zacks, 1979; Posner \& Snyder, 1975; Shiffrin \& Schneider, 1977). In their influential paper, Posner and Snyder laid out specific criteria for classifying a process as automatic, rather than as conscious. According to Posner and Snyder, an automatic process (1) occurs without intention, (2) is not open to conscious awareness or introspection, and (3) consumes few, if any, conscious resources. Conscious processes are ones that, by default, do not meet these criteria.

The idea that words automatically activate their meanings has brought forth two of the most heavily cited examples of automaticity: the Stroop effect (Stroop, 1935) and the semantic-priming effect (see Neely \& Kahan, 2001, for a review). Investigations of both effects have focused on Criteria 1 (intentionality) and 3 (resource independence). In the Stroop task, words such as red, green, blue, or yellow are presented visually to participants, but written in mismatching colors (e.g., the word green writ- ten in red ink). Participants, even though they are warned to ignore the words and respond only to the color, show drastic interference (see MacLeod, 1991, for a review). The semantic-priming effect refers to the common finding that responding to a target word, such as cat, is faster (in naming and lexical decision tasks) following a semantically related prime (e.g., $d o g$ ) than following an unrelated prime (e.g., table). Like the Stroop effect, the semanticpriming effect seems unavoidable, in that it occurs even when participants are consciously led to expect a target from an unrelated category (Neely, 1977).

As a result of semantic-priming studies, researchers have suggested that words automatically activate both their meanings and those of closely associated words in memory. This could occur through a spreading activation mechanism in which activation automatically spreads from the holistic semantic representation of a word to those of semantically associated neighbors (Neely, 1977; Posner \& Snyder, 1975). Another possibility is that reading a word (e.g., cat) activates semantic features associated with that word (e.g., fur, claws, four legs, etc.), which, in turn, facilitates the processing of other concepts (e.g., dog) sharing these features (Kawamoto, 1993; Masson, 1995; Moss, Hare, Day, \& Tyler, 1994; Plaut, 1995). Although there is much debate between spreading activation and feature overlap explanations of priming (see Hutchison, 2003, for a review), both theories assume automatic activation of the prime word's semantic representation (regardless of how the representation is implemented).

Interestingly, although both the Stroop effect and semantic priming are considered the hallmarks of automaticity, the intentionality and/or resource availability

K.A. Hutchison, khutch@montana.edu 
criteria have been called into question for both effects. Instead, these effects may depend on the level of representation at which people focus their attention. For instance, early semantic-priming studies by Smith (1979), Smith, Theodor, and Franklin (1983), and Henik, Friedrich, and Kellogg (1983) showed that semantic priming did not occur if participants searched the prime for a particular letter, arguing that attending to specific letters prevents activation of the word's meaning. Interestingly, performing a letter search appears to selectively eliminate the activation of semantics, sparing lexical and phonological activation. The effects of word identity (Friedrich, Henik, \& Tzelgov, 1991), lexicality (Reicher, 1969), phonology (Ziegler, Van Orden, \& Jacobs, 1997), and morphology (Stolz \& Besner, 1998) remain intact following letter search (but see Kahan, Sellinger, \& Broman-Fulks, 2006, for evidence that letter search eliminates priming from phonologically related words).

Besner, Stolz, and Boutilier (1997) used a manipulation similar to the letter search task to investigate the automaticity of semantic activation in the Stroop task. In this experiment, either all the letters of the incongruent word were colored, or only a single letter was colored (e.g., only the letter $l$ in blue). As was observed for semantic priming, Besner et al. (1997, Experiment 2) found that focusing attention on a single letter eliminated the Stroop effect. The focus of our initial discussion will be on the major theories regarding the prime task effect on semantic priming, since this has been more heavily researched. However, it should be noted that Besner and colleagues initially argued that the same process could explain the elimination of the Stroop effect.

\section{Current Models for the Prime Task Effect}

Pathway-blocking and resource-dependent activation. McClelland and Rumelhart's (1981) interactive activation model posited distinct letter, lexical, and semantic levels of representation, with excitatory connections between levels and inhibitory connections within levels. Borowsky and Besner (1993) demonstrated how this model can explain semantic priming if it is assumed that the prime word's lexical representation activates related concepts in the semantic level via between-level excitatory connections. Top-down feedback from the semantic level to the lexical and letter levels allows these related items to be more easily recognized. To explain the prime task effect, Stolz and Besner (1996) later argued that when attention is focused at the letter level, activation feeds forward from the letter to the lexical level but is blocked from reaching the semantic level. Specifically blocking the lexical-semantic pathway thus precludes the activation of any semantically related items but allows top-down activation from the lexical level to influence letter search performance.

The pathway-blocking hypothesis is elegantly simple in its ability to explain the prime task effect. However, additional results have rendered the theory more complicated. For instance, Henik, Friedrich, Tzelgov, and Tramer (1994) found significant (although still reduced) semantic priming following letter search when the relatedness proportion between primes and targets was high (80\%). This suggested that the lexical-semantic pathway may not be blocked if participants consider semantic information relevant. Moreover, further experiments have shown intact semantic priming following other, shallow prime tasks, such as color discrimination (Chiappe, Smith, \& Besner, 1996; MacNevin \& Besner, 2002) or number matching (Brown, Roberts, \& Besner, 2001). Stolz and Besner (1996, 1999) explained this pattern by suggesting that blocking the lexical-semantic pathway requires attention to be devoted specifically to another level within the word-processing domain. Other processing domains, such as judging colors or numbers, make few or no demands on the word recognition system.

In a later set of experiments, Stolz and Besner (1996) found that letter search eliminated semantic priming if the probe letters were presented simultaneously with the prime words, but not if they were delayed by $200 \mathrm{msec}$. However, when both simultaneous and delayed conditions were intermixed within the same block of trials, semantic priming occurred in both conditions. Thus, whether or not the lexical-semantic pathway is blocked may depend on the attentional set adopted by the participant.

A related model by Smith, Bentin, and Spalek (2001) states that the degree of semantic activation is dependent on the attentional resources devoted to the semantic versus the letter level. This resource-dependent activation model predicts that semantic activation is not an all-or-none process. In one experiment, Smith et al. (2001) asked participants to respond to primes, using a traditional letter search, a consonant/vowel decision on the first letter, or an animacy (living/nonliving) judgment. They obtained a 53-msec priming effect following the semantic-level animacy judgment. Although it was reduced following both letter tasks, there was a significant 24-msec priming effect following the easy consonant/vowel task and a nonsignificant 12-msec priming effect following the traditional letter search task. An additional experiment revealed that response times (RTs) to consonant/vowel judgments were much shorter than RTs to traditional letter search, suggesting that the consonant/vowel task required fewer attentional resources. These results more strongly support resource-dependent activation, rather than all-or-none activation. However, it should be noted that these models would become virtually indistinguishable if one assumed that blocking of the lexical-semantic pathway can occur in a probabilistic fashion depending on task demands.

Activation-based suppression. Keele and Neill (1978) argued that semantic representations are activated automatically whenever a stimulus is encountered; however, such representations are sometimes irrelevant or even incompatible to one's current task (e.g., the Stroop task). According to Keele and Neill, such irrelevant representations can be thought of as cognitive noise that must be suppressed in order to perform the task. Similar arguments have repeatedly resurfaced as an explanation for the phenomenon of negative priming, in which people are slower to respond to target items identical or semantically related to a previously ignored distractor (Houghton \& Tipper, 1994; Malley \& Strayer, 1995; Neill \& Westberry, 1987; Tipper, 1985; Tipper, Weaver, \& Houghton, 1994). 
In her review, Maxfield (1997) claimed that such an activation-based suppression model could explain the prime task effect. If the semantic representation of a word becomes activated during a letter search, it should be inhibited to avoid competition with the relevant letter information for control of attention. As a result, a lack of priming need not imply a lack of semantic activation, because priming paradigms must infer such processing on the basis of responses to a later target. Null priming could result from suppression that drives the irrelevant prime's semantic representation back to baseline levels prior to the onset of the target. (For further explanations of how inhibition can spread to related items, see Houghton \& Tipper, 1994, and Hutchison, 2002.)

In order to test for activation-based suppression, MaríBeffa, Fuentes, Catena, and Houghton (2000) combined the letter search and the negative-priming paradigms by presenting a word for letter search combined with a tobe-ignored distractor word. If attention indeed prevents or limits the activation of semantics (as is suggested by the pathway-blocking and resource-dependent activation models), there should be no priming from either the attended or the ignored word. Although Marí-Beffa et al. (2000) found no significant semantic priming, following letter search, from attended items, they found significant priming from distractor items. The opposite effect was observed when lexical decision was performed on the prime, with significant semantic priming from attended items and negative priming from ignored distractors. According to Marí-Beffa et al. (2000), the competing irrelevant information depends on the prime task. In a letter search task, the prime word's meaning competes for attention and must be suppressed. However, in lexical decision, the meaning of the prime word is relevant, whereas the distractor word's meaning must be suppressed.

Recent studies by Heil, Rolke, and Pecchinenda (2004) and Marí-Beffa, Valdés, Cullen, Catena, and Houghton (2005) in which event-related brain potentials (ERPs) were used also suggest that a prime word's meaning may be activated during letter search. These studies examined the N400, an ERP component peaking around $400 \mathrm{msec}$ after word onset that has previously been demonstrated to reflect semantic processing (Besson, Fischler, Boaz, \& Raney, 1992; Deacon, Hewitt, Yang, \& Nagata, 2000; Kiefer \& Spitzer, 2000; Osterhout \& Holcomb, 1995). In their RT analysis, Heil et al. observed the typical pattern of repetition, but not semantic, priming following letter search. However, Heil et al. found significant priming in the N400 amplitude for both repeated and semantically related targets, as compared with unrelated targets. MaríBeffa et al. (2005) recently failed to obtain N400 target priming following letter search but did find ERP and RT differences in responding to words representing living versus nonliving objects during the letter search task itself. This pattern implies that some degree of semantic activation occurred during letter search but did not facilitate later responding to the related target.

Recent Stroop and semantic-priming dissociations. As was discussed previously, Besner et al. (1997) origi- nally invoked the pathway-blocking model to explain the elimination of Stroop interference when only a single letter is colored. However, there is growing evidence that the reduction in priming from letter search and the reduction in the Stroop effect from single-letter coloring may be due to different mechanisms. For example, researchers have found either positive priming (Catena, Fuentes, \& Tudela, 2002) or negative priming (Besner, 2001) from stimuli producing no Stroop interference in the singleletter-colored condition. Such dissociations prompted MacNevin and Besner (2002) to test whether the singleletter coloring procedure used to eliminate Stroop interference would also eliminate semantic priming by asking participants to indicate the color of a single letter within each prime word. Interestingly, MacNevin and Besner (2002) obtained both semantic and morphemic priming using this procedure. As has been suggested by MacNevin and Besner, "it is possible that the onset of semantic processing in Stroop is slightly delayed [but not blocked] by coloring a single letter" (p. 115). Manwell, Roberts, and Besner (2004) later argued that cuing a single colored letter does not block semantic processing but, instead, makes it easier for participants to discriminate between relevant and irrelevant sources of activation.

\section{The Present Study}

MacNevin and Besner (2002) demonstrated that the single-letter coloring procedure eliminates the online measure of semantic activation (i.e., Stroop interference), but not the downstream measure of semantic activation (i.e., priming). This dissociation suggests that semantic activation may occur in the single-letter Stroop task. The present study was an attempt to address the second half of this potential double-dissociation - namely, does the letter search task eliminate the downstream measure (i.e., priming), but not the online measure (i.e., the Stroop effect)? The recent ERP research by Marí-Beffa et al. (2005) indeed suggests that one could obtain evidence for semantic processing during letter search in the absence of semantic priming. To test this question, we included words in the letter search task whose meanings should bias one of the two possible decisions (e.g., the word present or the word $a b s e n t$ ). If the meaning of the word is indeed activated during letter search, we should observe a semantic congruency effect: faster or more accurate responding when the meaning of the word matches the appropriate response (e.g., searching for the letter $s$ in present) than during a mismatch (e.g., searching for $s$ in absent). In Experiment 1 , the participants performed a letter search on positive bias words (e.g., present), negative bias words (e.g., absent), and neutral words (e.g., liberty). In Experiment 2, the positive and negative bias words were paired with unrelated targets and were included along with semantically related and unrelated prime-target pairs. The critical question was (1) whether these critical items would show a semantic congruency effect in the letter search task and, if so, (2) whether this congruency effect would remain under conditions in which semantic priming had been eliminated. 


\section{EXPERIMENT 1}

\section{Method}

\section{Participants}

Eighteen undergraduates at Montana State University participated for partial completion of a research requirement for an introductory psychology class. All were native English speakers with normal or corrected-to-normal vision.

\section{Stimuli}

Each trial consisted of a target word with a probe letter above it. As is standard in letter search experiments (Besner, Smith, \& MacLeod, 1990; Henik et al., 1994), the probe letter appeared above every letter in the prime word, as shown below:

\section{eeeeeee$$
\text { liberty }
$$

On half of the trials, the probe letter occurred in the target word, and on half of the trials, it did not. For positive trials, the probe letter was drawn equally often from the beginning, middle, and end of the target word. The stimuli and probe letters selected are presented in the Appendix. To make negative trials, the probe letters from the positive trials were re-paired with other words, so that any given probe letter appeared equally often in positive and negative conditions. This counterbalancing prohibited participants from using the identity of the probe letter to bias a present/absent response.

A total of 24 target words were used in the experiment. Each target word was shown three times in the letter-present condition (with the probe letter taken from one of the beginning, middle, or end positions) and three times in the letter-absent condition. Twelve of the items were bias words, and 12 were neutral words. For the bias items, 6 had meanings that were related to a positive response in the letter search task, and 6 had meanings related to a negative response. The bias words chosen were contain, identical, match, present, same, yes, absent, different, lacking, mismatch, missing, and no. The average position of the probe letter within the word was 3.6 $(S D=1.7)$ for positive words and $3.7(S D=2.1)$ for negative words ( $t<1$ for the difference in probe letter position between positive and negative words). In addition, the positive and negative bias words had mean lengths of 5.8 and 6.5 letters, respectively, and mean $\log$ HAL frequencies of 11.0 and 10.1. Neither of these differences was significant (both $p \mathrm{~s}>.40$ ).

In addition to the 12 critical words, we also generated 12 responseneutral words (e.g., butter) that were equated in length with the critical items (6.2 and 6.3 letters for the critical and neutral words, respectively; $p>.80)$. Further analyses revealed that the responseneutral items had lower log HAL frequencies than did the critical items [9.2 vs. $10.5 ; t(22)=2.15, p<.05]$.

\section{Procedure}

The participants were tested individually and were seated approximately $60 \mathrm{~cm}$ from a VGA monitor; they read a set of task instructions displayed on the monitor and then heard them paraphrased by the experimenter. The participants were instructed that they would see a word with a probe letter repeated above it and that they should indicate whether or not the letter was contained in the word. They were told to press the " $p$ " key if the letter was contained in the word or to press the "q" key if the letter was absent. Each trial contained the following events: a 1,000-msec fixation point (*), a 500-msec interstimulus interval, and the target word and letter probe. All the stimuli were presented centered on the display monitor. A 1,000-msec blank screen interval preceded each new trial. The participants were given 6 practice trials using new neutral stimuli, with three of the words in the letter-present condition and three in the letter-absent condition. After the practice trials, the participants were asked whether they had any questions and were told to begin the experiment. The participants were asked to make their response as quickly and accurately as possible on all the experimental trials.
The participants responded to a total of 144 experimental trials, with self-paced rest breaks given every 36 trials.

\section{Design}

There were two within-subjects variables in the design: bias (neutral words, negative words, or positive words) and letter presence (letter present or letter absent). The participants' response latencies and error rates were examined in each of the conditions above. However, due to a programming error, no error data were recorded for 3 of the participants.

\section{Results}

Only correct responses were considered for the RT analysis. In this and all further analyses, we used the modified recursive outlier removal procedure proposed by Van Selst and Jolicœur (1994). This procedure removed 1.9\% of the correct RTs. Mean response latency and percentage of errors were calculated for each participant for each cell. The bias effect was computed by subtracting the mean RT or the percentage of errors to positive bias words from that to negative bias words. These data are presented in the top of Table 1. Unless otherwise noted, each effect called statistically significant is associated with a two-tailed $p<.05$.

RTs and percentages of errors for the six conditions were submitted to ANOVAs with both bias and letter presence varied within subjects. There was a significant effect of letter presence $\left[F(1,17)=26.60, M S_{\mathrm{e}}=8,369\right]$. The participants were $91 \pm 37 \mathrm{msec}$ faster to respond on letter-present trials than on letter-absent trials. (When an effect of $X \pm Y \mathrm{msec}$ is reported, $Y$ refers to the $95 \%$ confidence interval.) This effect replicates the results of previous letter search experiments (Besner et al., 1990; Smith, 1979; Stolz \& Besner, 1996) and makes intuitive sense if one assumes that detection of the letter allows the participant to respond immediately, without having to search the rest of the word. In addition, there was a main effect of bias $\left[F(2,34)=9.81, M S_{\mathrm{e}}=5,398\right]$. The participants responded $94 \pm 62 \mathrm{msec}$ more quickly to neutral words than to negative words and $78 \pm 47 \mathrm{msec}$ more quickly to positive words than to negative words. Response latencies to

Table 1

Response Latencies (in Milliseconds) and Percentages of Errors $(\%$ E) to Neutral, Negative, and Positive Bias Words in the Letter Search Task in Experiments 1 and 2

\begin{tabular}{|c|c|c|c|c|}
\hline \multirow[b]{3}{*}{ Word Meaning } & \multicolumn{4}{|c|}{ Response Condition } \\
\hline & \multicolumn{2}{|c|}{ Letter Present } & \multicolumn{2}{|c|}{ Letter Absent } \\
\hline & RT & $\% \mathrm{E}$ & RT & $\% \mathrm{E}$ \\
\hline \multicolumn{5}{|c|}{ Experiment 1} \\
\hline Neutral & 1,033 & 4.2 & 1,127 & 2.0 \\
\hline Negative & 1,151 & 10.7 & 1,198 & 1.9 \\
\hline Positive & 1,030 & 8.2 & 1,162 & 3.0 \\
\hline Negative - positive & $+120^{*}$ & +2.5 & +36 & -1.1 \\
\hline \multicolumn{5}{|c|}{ Experiment 2} \\
\hline Neutral & 1,039 & 7.5 & 1,085 & 4.6 \\
\hline Negative & 1,096 & 8.9 & 1,146 & 4.7 \\
\hline Positive & 1,021 & 5.7 & 1,124 & 3.9 \\
\hline Negative - positive & $+74^{*}$ & $+3.2^{*}$ & +21 & +0.8 \\
\hline
\end{tabular}

Note-For Experiment 2, neutral means are collapsed across repeated and nonrepeated items. ${ }^{*} p<.05$. 
neutral and positive words did not differ $(16 \pm 46 \mathrm{msec})$. Of most importance, the two-way bias $\times$ letter presence interaction was marginally significant $[F(2,34)=2.81$, $\left.M S_{\mathrm{e}}=5,687\right]$. In the letter-present condition, people responded more quickly to both the positive bias words $(120 \pm 63 \mathrm{msec})$ and the neutral words $(117 \pm 66 \mathrm{msec})$ than to the negative bias words. In the letter-absent condition, people still responded marginally more quickly (70 \pm $76 \mathrm{msec}$ ) to the neutral words than to the negative words. However, there was now no difference in RTs between the positive and the negative words ( $36 \pm 57 \mathrm{msec})$.

A second 2 (bias) $\times 2$ (letter presence) ANOVA was run on only the two critical bias conditions (positive vs. negative). This ANOVA revealed not only the two main effects reported above, but also a significant two-way interaction $\left[F(1,17)=5.56, M S_{\mathrm{e}}=5,715\right]$, revealing that the bias effect in the letter-present condition was significantly different from the nonsignificant bias effect in the letter-absent condition. As was discussed previously, this interaction reflects the influence of congruency between the meaning of the words and the appropriate response, so that the mean RT for the two congruent items (letterpresent/positive word and letter-absent/negative word) was $42 \pm 38 \mathrm{msec}$ shorter than the mean RT for the two incongruent items (letter-present/negative word and letterabsent/positive word). ${ }^{1}$

The error rate was relatively low, with an overall mean of $5.0 \%$. There was a significant effect of letter presence $\left[F(1,14)=20.78, M S_{\mathrm{e}}=31.94\right]$. The participants made $5.4 \% \pm 2.6 \%$ more errors in the letter-present condition than in the letter-absent condition. This effect replicates the results of previous research (Besner et al., 1990; Brown et al., 2001; Smith, 1979) and indicates that the participants were more likely to miss detecting a letter that was present than they were to detect a letter that was absent. In addition, a main effect of bias was obtained $[F(2,28)=$ $\left.7.21, M S_{\mathrm{e}}=11.28\right]$. The participants made $3.1 \% \pm 1.9 \%$ fewer errors to the neutral words than to the negative words and $2.4 \% \pm 1.5 \%$ fewer errors to the neutral words than to the positive words. Finally, the two-way bias $\times$ letter presence interaction was significant $\left[F(2,28)=5.81, M S_{\mathrm{e}}=\right.$ 14.41]. This interaction revealed that the presence effect (more errors in the letter-present condition), although significant for all three classes of words, may have been different for the neutral $(2.2 \% \pm 2.1 \%)$, positive $(5.2 \% \pm$ $4.4 \%)$, and negative $(8.9 \% \pm 3.6 \%)$ items.

A second 2 (bias) $\times 2$ (letter presence) ANOVA was run on only the two critical bias conditions (positive vs. negative). This ANOVA revealed only a main effect of letter presence $\left[F(1,14)=21.15, M S_{\mathrm{e}}=35.12\right]$, with participants making more errors in the letter-present condition. The two-way interaction with bias did not reach significance $(p>.11)$, although it was in the predicted direction, so that people tended to make fewer errors on absent trials and more errors on present trials when the word was negative.

\section{Discussion}

These data provide evidence for automatic activation of meaning in a letter search task. Specifically, we ob- tained a congruency interaction between the meaning of the target word (positive or negative) and the appropriate response (present or absent). This congruency effect occurred in conjunction with a general tendency to respond more slowly to negative items. The resulting pattern indicated that although people were faster to respond to positive than to negative words in both letter-present and letter-absent conditions, congruent items (negativeword/letter-absent and positive-word/letter-present) were responded to more quickly and more accurately, on average, than were incongruent items (negative-word/letterabsent and positive-word/letter-present). In other words, the participants were especially fast at responding present when the word was positive, rather than negative. For error rates, the participants were especially likely to miss a letter when the word was negative. The fact that word meaning interacted with the presence judgment provides compelling evidence that the meaning of the word was activated in the letter search task. Thus, these results argue against the pathway-blocking and resource-dependent activation models.

The main effect of bias, in which the participants responded more slowly to negative words than to positive or neutral words, prevented the data from showing a complete crossover interaction in which RTs are determined solely by the match between the meaning of the word and the required response. Although the two sets of words were equated on length and frequency, it was not possible to equate them on all possible variables. Indeed, a post hoc examination of the items revealed that six of the negative words are inflected (i.e., have a prefix or suffix), whereas none of the positive words are. Furthermore, all of the negative words are lexically marked, whereas all of the positive words are unmarked (Greenberg, 1966, 1987). Any difference in search difficulty between item sets could explain not only the general slowdown for the negative words, but also, perhaps, the overall congruency effect, in that increased difficulty of searching the negative bias words could favor no responses and hinder yes responses to the negative bias words.

However, there are problems for search difficulty as an alternative explanation for the present data. First, if participants perform a serial exhaustive search through the word, the effect of search difficulty should be larger the more letters there are that need to be searched (i.e., the absent condition). Therefore, contrary to the observed pattern, this explanation predicts that the difference between positive and negative words should have been larger for the letterabsent condition than for the letter-present condition. The second problem for this alternative theory is that it would predict an interaction between negative and neutral words as well. Recall that both the neutral and the positive words were responded to more quickly than were the negative words in the letter-present condition, yet only responses to the neutral words continued to be (marginally) faster than those to the negative words in the letter-absent condition. This pattern is more consistent with the congruency explanation than with the search difficulty explanation. A possible alternative explanation is that negative valiance words produce an overall delay in responding (see Algom, 
Chajut, \& Lev, 2004, for a review). This possibility will be addressed following the discussion of Experiment 2.

The present study also showed a letter presence effect in both RTs and error rates. The participants responded more quickly in the letter-present condition but also made more errors in that condition. The faster responding was most likely driven by the participants' stopping their search through the letter string once a match was obtained. The higher error rate likely occurred because it was easier to miss an item that was present than to "see" an item that was not. However, although these effects are common in the letter search literature, response hand was not counterbalanced in Experiment 1, so these explanations may be premature.

As was discussed above, the most plausible explanation of Experiment 1 is that the RTs and errors were influenced by the congruency between the meaning of the to-be-searched stimulus and the required response. However, there are still three concerns regarding Experiment 1 that prevent drawing any firm conclusions pertaining to the activation of meaning in other letter search tasks. The first concern is the possibility that the participants had not received enough practice learning to suppress word meanings in the service of letter matching. With only six practice trials, perhaps the congruency effect was driven primarily by participants who had not yet learned to focus on the letter level and block the activation of meaning. The second concern is that the words were presented six times each. Perhaps ignoring a word becomes more difficult across repetitions (but see Henik et al., 1994, for a demonstration that letter search eliminates priming even with repeated items). The third problem is that $50 \%$ of the stimuli in Experiment 1 were related to the concepts of presence and absence. Such a high proportion of related items may have made these semantic categories more salient and, therefore, prevented the participants from blocking the activation of meaning (see Stolz \& Besner, 1996).

\section{EXPERIMENT 2}

The concerns above were addressed in Experiment 2 in three ways. First, we provided the participants with 30 practice trials prior to the start of the letter search task. This should allow them sufficient time to learn how to focus their attention at the letter level, rather than at the word level. Second, we presented each of our bias items two times (once in the letter-present and once in the letter-absent conditions), rather than six times, to reduce the salience of the semantic categories of presence and absence. This was done knowing that it might weaken the strength of our manipulation, since such a reduction in repetitions led to only 6 observations in each of the critical bias $\times$ letter presence cells (as compared with 18 in Experiment 1). Third, 36 neutral primes (12 shown twice, 24 shown once) were presented in the letter search task, rather than 12 . As a result, only $25 \%$ of the letter search words in Experiment 2 were related to the concepts of presence/absence. Finally and most important,
Experiment 2 employed a prime task procedure in which the participants received both prime and target items and generated a speeded naming response to the targets and either a speeded naming or a letter search response to the primes. The bias stimuli were embedded within the letter search trials and served as "catch" trials to detect whether the meanings of to-be-searched words were being activated during the letter search task. We expected to replicate the typical prime task effect (i.e., significant priming following named primes and reduced or eliminated priming following letter-searched primes). The critical prediction concerned the possibility of a congruency effect when priming is eliminated. If, indeed, the prime task effect is caused by the elimination of semantic activation during letter search, we should see no congruency effect. However, if, instead, the prime task effect is caused by something that occurs following semantic activation, we should replicate the congruency effect found in Experiment 1 even in the absence of priming.

\section{Method}

\section{Participants}

One hundred twenty-eight undergraduates at Montana State University participated for partial completion of a research requirement for an introductory psychology class. All were native English speakers with normal or corrected-to-normal vision. The data from 5 participants were eliminated due to error rates greater than $30 \%$ in the letter search task. Their data were replaced by those from 5 additional participants in order to complete the necessary counterbalancing.

\section{Stimuli}

Bias stimuli. The same bias words as those in Experiment 1 were used, with one exception: The word mismatch was replaced with the word omitted. (This was done because we were concerned that the base morpheme match may have caused a positive, rather than a negative, response bias.) Each bias word was presented twice: once in the letter-present condition and once in the letter-absent condition. In the letter-present condition, 2 of the 12 stimuli had the letter selected from the beginning of the word, 2 from the middle of the word, and 2 from the end of the word. The average position of the probe letter was $2.5(S D=1.4)$ for positive words and $3.3(S D=$ 2.2) for negative words ( $t<1$ for the difference in probe letter position between positive and negative words). As in Experiment 1, negative trials were created by re-pairing letters from positive trials. The neutral words in Experiment 1 were not used in Experiment 2. (See the Appendix for a list of the bias stimuli and probe letters chosen for Experiment 2.)

For each of the bias words, we generated two unrelated words to serve as targets for a pronunciation response. These words had a mean length of 4.9 letters and a mean log HAL frequency of 10.3 . These items also had average naming latencies of $605 \mathrm{msec}$ from the English Lexicon Project (ELP) database (Balota et al., in press), which contains lexical decision and naming data for over 40,481 single-syllable English words.

Neutral stimuli. We generated 72 strongly associated primetarget pairs, using the Nelson, McEvoy, and Schreiber (1999) word association norms, so that when a person was presented with the prime word, the word with the highest probability of being generated as an associate was selected as the target. Forty-eight of these pairs were chosen to serve as our nonrepeated prime-target pairs, and 24 were chosen to serve as our repeated pairs. The repeated and nonrepeated pairs were included to demonstrate that the prime task effect generalizes to both sets of items. If such a pattern were to be 
found, this would preclude any arguments that repeating the bias words made their meanings more strongly activated (and therefore, less likely to be "blocked") than the neutral items. This led to a total of 96 neutral trials. The Nelson et al. forward associative strengths were .68 and .69 for the nonrepeated and repeated pairs, respectively, and the backward associative strengths were .31 and .30 . The repeated and nonrepeated primes and targets were also equated in length, frequency, and ELP naming latency (all $p s>.15$ ). For the nonrepeated items, half of the prime-target pairs appeared in the related condition and half appeared in the unrelated condition. The repeated pairs were shown once in the related and once in the unrelated conditions. Unrelated pairs for both repeated and nonrepeated items were created by re-pairing the primes and the targets. The nonrepeated primes appeared in either the letter-present or the letter-absent condition (repeated primes appeared once in each). As with the bias stimuli, the probe letters used in the letter-present condition were equally likely to be taken from the beginning, middle, or end of the word, and negative trials were created by re-pairing letters from positive trials. The neutral targets did not differ from the bias targets in length, frequency, or ELP naming latency (all $p \mathrm{~s}>.25)$. However, the neutral primes were shorter than the bias primes (5.1 vs. 6.1, respectively) and less frequent (9.0 vs. 10.6; both $p \mathrm{~s}<.05$ ). In addition to counterbalancing the prime-target pairs across letter presence and relatedness conditions, pairs were also counterbalanced across task, with half of the pairs presented in the pronunciation task and half in the letter search task. This led to a total of eight different stimulus lists.

\section{Procedure}

The participants were tested individually and were seated approximately $60 \mathrm{~cm}$ from a VGA monitor; they read a set of task instructions displayed on the monitor and then heard them paraphrased by the experimenter. The participants were instructed that there were two sections to the experiment. The order of the two prime tasks (letter search vs. pronunciation) was counterbalanced across participants. The procedure for the letter search task was the same as that in Experiment 1, with three exceptions. First, the response hand was counterbalanced across participants, so that half were told to press the "p" key if the letter was contained in the word and to press the "q" key if the letter was absent and the other half received the opposite instructions. Next, the participants received a total of 102 trials ( 30 practice and 72 experimental). Finally, the participants were told to pronounce the target word, which appeared $600 \mathrm{msec}$ after their prime response, as quickly and accurately as possible. After the participants' pronunciation response, a 1,000-msec intertribal interval blank screen preceded the next trial.

The presentation of stimuli for the pronunciation task mimicked the letter search task. The participants were told to pronounce the word stimulus on both the prime and the target trials. The participants responded to a total of 78 pronunciation trials (30 practice and 48 experimental). (Note that the absence of the 12 repeated bias words in the pronunciation task reduced the total number of experimental trials by 24 . This was necessary to prevent having to present each bias word four times in the experiment.) The entire session lasted approximately $30 \mathrm{~min}$, with the participants responding in a total of 180 trials (60 practice and 120 experimental) across the two tasks. Self-paced rest breaks were given approximately every 30 trials.

\section{Design}

Participants performed both the letter search task and the pronunciation task. For each stimulus list, half of the prime-target pairs were shown once in either the related or the unrelated condition and the other half were shown once in each condition. The bias words used in the letter search task were always presented twice. In both the pronunciation and the letter search tasks, the prime words were presented in either the letter-present or the letter-absent condition. This led to a 2 (task) $\times 2$ (letter presence) $\times 4$ (item type: nonrepeated neutral, repeated neutral, positive bias, or negative bias) within-subjects design.

\section{Results}

\section{Prime Responses}

The participants performed either a letter search or pronunciation on prime words. Incorrect responses in the letter search task and blatant microphone errors in the pronunciation task $(\mathrm{RT}=0)$ were eliminated prior to analyzing the RT data. The recursive outlier procedure removed $1.8 \%$ and $2.4 \%$ of the remaining letter search and naming trials, respectively.

Response-neutral primes. Mean response latency was calculated for each participant in each of the eight cells created by the prime task $\times$ letter presence $\times$ repetition conditions. RTs and percentages of errors were submitted to a within-subjects ANOVA. There was a significant effect of task $\left[F(1,127)=597.37, M S_{\mathrm{e}}=130,759\right]$, with the participants responding $552 \pm 44 \mathrm{msec}$ more quickly when pronouncing primes than when performing a letter search on them. There was also a task $\times$ letter presence interaction $\left[F(1,127)=20.38, M S_{\mathrm{e}}=7,668\right]$. This interaction was caused by the participants' responding $46 \pm$ $22 \mathrm{msec}$ more quickly to letter-present trials than to letterabsent trials in the letter search task but a nonsignificant $3 \pm 7 \mathrm{msec}$ slower in the pronunciation task. Thus, the presence or absence of the probe letter in the prime below it influenced responding in the letter search task but had no effect on responding in the pronunciation task. No other effects approached significance.

A 2 (repetition) $\times 2$ (letter presence) ANOVA was used to examine letter search accuracy. As in Experiment 1, there was a significant effect of letter presence $[F(1,127)=$ $\left.22.31, M S_{\mathrm{e}}=50.35\right]$. The participants made $3.0 \% \pm 1.2 \%$ more errors in the letter-present condition than in the letterabsent condition. In addition, a main effect of repetition was obtained $\left[F(1,127)=14.47, M S_{\mathrm{e}}=61.48\right]$. The participants made $2.6 \% \pm 1.4 \%$ more errors to repeated prime words. Finally, the interaction between repetition and letter presence did not approach significance $(F<1)$.

Bias primes. In order to test for a congruency effect in the letter search task for Experiment 2, we conducted a 2 (letter presence) $\times 2$ (bias) ANOVA on both RTs and errors. The means and percentages of errors for these conditions are shown at the bottom of Table 1 . In the RTs, there was an effect of letter presence $[F(1,127)=$ 26.96, $M S_{\mathrm{e}}=27,999$ ], with the participants responding $77 \pm 29 \mathrm{msec}$ more quickly on the letter-present trials than on the letter-absent trials. In addition, there was a main effect of bias $\left[F(1,127)=20.07, M S_{\mathrm{e}}=14,852\right]$. The participants responded $48 \pm 21 \mathrm{msec}$ more quickly to positive words than to negative words. Of most importance, the two-way bias $\times$ letter presence interaction was significant $\left[F(1,127)=6.26, M S_{\mathrm{e}}=13.667\right]$. The bias effect (decreased latency in responding to positive words, relative to negative words) was significantly greater for letter-present responses (a significant $74 \pm 32 \mathrm{msec}$ effect) than for letter-absent responses (a nonsignificant $21 \pm 27 \mathrm{msec}$ effect). Thus, the congruency between the meaning of the words and the appropriate response again influenced responding, so that the mean RT for the two congruent conditions (letter-present/positive-word and 
letter-absent/negative-word) was $26 \pm 20 \mathrm{msec}$ shorter than the mean RT for the two incongruent items (letterpresent/negative-word and letter-absent/positive-word).

A second ANOVA run on error rates revealed an effect of letter presence $\left[F(1,127)=10.74, M S_{\mathrm{e}}=106.88\right]$, with participants making more errors in the letter-present condition, and an effect of bias, with participants making more errors to negative words. The two-way interaction with bias did not reach significance $(p>.14)$, although it was again in the predicted direction based on response congruency, so that participants made $3.1 \% \pm 2.2 \%$ fewer errors on letter-present trials when the word was positive than when the word was negative but showed no significant difference in error rates on letter-absent trials.

\section{Target Responses}

Trimming of RTs was done in the same manner as that for prime responses. Pronunciation responses of $0 \mathrm{msec}$ (microphone errors) or responses following letter search errors were eliminated prior to analyzing the data (such outliers accounted for $5.2 \%$ of overall target pronunciations). Next, the modified recursive outlier removal procedure was used $(2.7 \%$ of the remaining target pronunciations were trimmed in this way).

Pronunciation latencies were submitted to a three-way ANOVA to evaluate the effects of prime task, repetition, and relatedness. The means for each of these conditions are shown in Table 2. Target pronunciation was $49 \pm$ $11 \mathrm{msec}$ faster when the prime was named than when it was searched for a letter $\left[F(1,127)=73.081, M S_{\mathrm{e}}=\right.$ $8,298]$, and $9 \pm 5 \mathrm{msec}$ faster for the repeated words than for the nonrepeated words $\left[F(1,127)=11.10, M S_{\mathrm{e}}=\right.$ $1,914]$. There was also a significant main effect of relatedness $\left[F(1,127)=15.70, M S_{\mathrm{e}}=1,359\right]$, with participants responding $9 \pm 5 \mathrm{msec}$ more quickly on related trials than on unrelated trials. Of most importance, this relatedness effect interacted with prime task $\left[F(1,127)=5.97, M S_{\mathrm{e}}=\right.$ $1,056]$, indicating that the letter search task eliminated semantic priming. The participants showed a significant $14 \pm 4 \mathrm{msec}$ relatedness effect when the primes were named but a nonsignificant $2 \pm 6 \mathrm{msec}$ relatedness effect when the primes were searched for a letter. No other effects approached significance (all $F \mathrm{~s}<1$ ).

Table 2

Naming Latencies to Target Stimuli (in Milliseconds) Following Either a Letter Search or Pronunciation of the Prime in Experiment 2

\begin{tabular}{lcc}
\hline & \multicolumn{2}{c}{ Prime Task } \\
\cline { 2 - 3 } Stimulus Condition & Letter Search & Naming \\
\hline Nonrepeated prime & 512 & 468 \\
Unrelated & 510 & 454 \\
Related & +02 & $+14^{*}$ \\
$\quad$ Priming & 504 & 461 \\
Repeated prime & 501 & 446 \\
Unrelated & +03 & $+15^{*}$ \\
Related & & \\
$\quad$ Priming & & \\
${ }^{*} p<.05$. & &
\end{tabular}

An additional analysis was conducted to examine the possibility that either prime length or prime frequency might moderate semantic priming following the letter search task. This was done because the bias primes used to detect the presence of a congruency effect were about one letter longer and slightly more frequent than the primes used to detect relatedness effects. However, further analyses revealed that priming effects following letter search did not significantly correlate with either prime length or prime frequency (both $p \mathrm{~s}>.50$ ). Priming following letter search was then computed separately for long and short primes (greater than or less than five letters) and for high- and low-frequency primes (log HAL frequencies of greater than 9.7 or less than 8.1 ). These values were chosen to compare the upper and bottom thirds of the items on each variable. In two separate ANOVAs, neither length nor frequency interacted with relatedness following letter search on the prime (both $F_{\mathbf{s}}<1$ ). Thus, we can be confident that differences in prime length and/or prime frequency did not artifactually produce the elimination of priming following letter search.

\section{Discussion}

The critical result of Experiment 2 was a replication of the congruency effect in Experiment 1 under conditions in which semantic priming was eliminated. The significant $14 \mathrm{msec}$ of priming that was obtained following pronunciation was reduced to a nonsignificant $2 \mathrm{msec}$ of priming following letter search. As was indicated previously, this pattern has traditionally been explained as the elimination of semantic activation when one's attention is focused on the letter level during the prime task. However, this interpretation is clearly incorrect, since we obtained a significant congruency effect from bias primes randomly presented within the same block of trials. The participants had an easier time responding to congruent stimuli in the letter search task than to incongruent stimuli. In addition, further analyses revealed that priming following letter search was eliminated regardless of the frequency or length of the primes and regardless of whether the primes were shown once or twice. This preempts possible arguments concerning methodological or item differences between the bias and the neutral primes. Finally, demonstrating both the letter search congruency effect and prime task effect in the same experiment preempts a possible criticism of Experiment 1 that inclusion of categorically related bias primes changed the participants' attentional set in such a way that they no longer blocked the activation of semantics (see Stolz \& Besner, 1996, for a discussion). If the inclusion of such items had caused the participants to pay attention to semantics, semantic priming should have been observed in the letter search task as well. Instead, our results suggest that the elimination of priming following letter search is due to processes that work after the initial activation of the prime word's semantic representation. Overall, these results are counter to predictions made by the pathway-blocking and resource-dependent activation models discussed in the introduction. 
Although the present methodology allowed us to rule out the criticism that including bias primes prevented the overall blocking of semantics, a possible limitation in this study is that we had to infer the presence of semantic activation in our neutral stimuli from the congruency effect obtained from our bias stimuli. Using different stimuli to measure congruency and priming could present a problem, since it is possible that semantic activation was occurring for our randomly intermixed bias primes, but not for the other neutral items. However, it probably would have been impossible to select items related to one bias prime but not to the others. In addition, each prime would have had to have been shown eight times to obtain just six critical trials in each of the task $\times$ relatedness $X$ letter presence conditions. In contrast, the use of catch trials has a long history in psychology. If the trials are randomly intermixed, it is reasonable to assume that people are not doing something different on catch trials than they are on the other trials. Indeed, previous research has demonstrated that the attentional set to block or not block semantic activation applies at a list-wide, rather than at an item-by-item, level (Stolz \& Besner, 1996). The most parsimonious explanation for the present data is, therefore, that the congruency manipulation is more sensitive to semantic activation than is priming. Additional support for this claim has come from the previously discussed Marí-Beffa et al. (2005) study, in which an RT effect of the prime's semantic category (living vs. nonliving) occurred in a letter search task, in association with a loss of semantic priming from these same primes.

Another possible limitation of Experiment 2 is that we once again obtained the congruency effect in conjunction with a general slowdown for negative bias words. As with Experiment 1, this prevented a complete crossover interaction, because responding to positive words was still numerically, but not significantly, faster than that to negative words even in the letter-absent condition. However, an inspection of the bottom of Table 1 does reveal a crossover interaction between the positive bias words and the neutral items. Indeed, an ANOVA comparing neutral and positive words in the letter-present and letter-absent conditions did reveal an interaction between letter presence and word type $[F(1,127)=11.87$, $\left.M S_{\mathrm{e}}=8,687\right]$. Responses to the positive words were numerically $18 \pm 22 \mathrm{msec}$ faster than those to the neutral items in the letter-present condition, yet $40 \pm 23 \mathrm{msec}$ slower than those to the neutral items in the letter-absent condition. Of importance, this crossover interaction between positive and neutral items occurs in the presence of no overall difference in RT for these items (1,062 and $1,073 \mathrm{msec}$ for the overall RT for neutral and positive items, respectively; $p>.17$ ), further supporting our claim that performance in the letter search task was influenced by the match between the meaning of the word and the required response.

\section{GENERAL DISCUSSION}

The present experiments demonstrate that semantic activation does occur in the letter search task. In Experi- ment 1 , the meaning of the to-be-searched word influenced the response given to that word, so that the participants were faster and more accurate at searching for the letter $s$ in the word present than in the word absent. In Experiment 2, we replicated the congruency effect obtained in Experiment 1 for our positive and negative bias words when they were randomly mixed with 48 neutral words that preceded related or unrelated targets in a pronunciation task. This congruency effect occurred in the absence of semantic priming, suggesting that our online measure of semantic activation (i.e., the congruency effect) was more sensitive than the downstream inference of semantic activation typically made in semantic-priming studies. When combined with MacNevin and Besner's (2002) results, a double dissociation is produced, with the single-letter coloring procedure affecting only the online measure of semantic activation (but not the downstream measure) and the letter search procedure affecting only the downstream measure of semantic activation (but not the online measure). This suggests that (1) these two procedures affect different processes and (2) neither procedure provides reasonable evidence against the automatic activation of meaning.

In both experiments, this letter search congruency effect appeared in conjunction with a general slowdown for negative words. Although the items were closely matched in printed word frequency, length, and probe letter position, further analysis using the ELP database (Balota et al., in press) indicated that people were numerically $55 \pm 75 \mathrm{msec}$ slower to make a lexical decision to the negative words used in the present experiment than to the positive words. This could explain the difference in overall letter search RT, since this measure correlated .27 $(p<.02)$ with letter search RT across all the items in the experiment. Another possibility is that negative-valenced words automatically capture attention, delaying responses in any task. A large literature on the emotional Stroop effect has demonstrated such a pattern, with slower responses across tasks to emotionally negative words (see Algom et al., 2004, for a review). However, such experiments typically use items such as danger or cancer that would likely be perceived as more threatening than the present negative words, such as absent or omitted. Nonetheless, this possibility is intriguing and could also explain an overall effect of bias. More research will be needed to determine whether mildly negative words still produce a slowdown in RT even when all known lexical and sublexical item characteristics are matched or statistically partialled out.

\section{Three Models Revisited}

The pathway-blocking and resource-dependent activation theories argue that the prime task effect is due to the elimination of semantic activation in the letter search task, either because the semantic pathway is blocked or because the resources needed to activate semantics is devoted to a difficult letter task. Our present results clearly run counter to these theories and, instead, suggest that whatever process led to the elimination of priming following letter search occurred after the initial activation of the prime. 
In contrast, our results are consistent with the activation-based suppression model of prime task effects proposed by Maxfield (1997) and Marí-Beffa and colleagues (Marí-Beffa, Fuentes, et al., 2000; Marí-Beffa, Houghton, Estévez, \& Fuentes, 2000). According to this model, all levels of representation (e.g., letter, word, and semantic) are automatically activated for prime words, regardless of whether people simply read the words or search the words for a particular letter. However, depending on one's goal (e.g., reading the word for comprehension vs. performing a letter search), some activated representations may cause interference and need to be suppressed. Therefore, this theory posits that semantic activation is indeed automatic (according to the intentionality, introspection, and resource independence criteria discussed in the introduction) but that conscious processes can lead to the suppression of such representations while an attempt is made to select an appropriate prime response. This theory can easily account for the present results by suggesting that the meanings of both the neutral and the bias words were activated during the letter search task. However, in order to select the appropriate prime response, these representations were suppressed. This suppression could then account for the failure to observe semantic priming from these items. An interesting question for future research concerns whether this suppression is greater for bias words (which should create an additional Stroop-like interference) than for neutral words (which should produce only a general word vs. letter interference). Clearly, the activation-based suppression model would predict such a difference. (Note that retrieval-based models, such as the episodic retrieval [Neill, Valdes, Terry, \& Gorfein, 1992] and temporal discriminability [Milliken, Joordens, Merikle, \& Seiffert, 1998] hypotheses, also make this prediction and will be discussed below.)

Although the present results are consistent with the activation-based suppression model, the previously discussed experiment by Heil et al. (2004) raises problems for this model. Recall that Heil et al. observed no semantic priming in RTs following letter search yet did find significant priming in the N400 effect. The critical question for those positing activation-based suppression is the following: Why would a suppressed representation cause an N400 priming effect?

A possible explanation for such a pattern is that participants retrieve a memory trace of prime episodes while processing the target (Neill, 1997; Neill \& Mathis, 1998; Neill et al., 1992; Tipper, 2001). According to such models, an ignored irrelevant prime stimulus can lay down a long-term memory trace of the processing it received during selection of the attended object (or level of representation within the object). Moreover, retrieval of past processing episodes involving similar stimuli reinstates comparable processing of an item in the present instance. According to Neill and Mathis, retrieving how a stimulus was previously processed can either help (transferappropriate processing) or hurt (transfer-inappropriate processing) responding to the current stimulus, depending on whether it is compatible with current goals. Simi- larly, Tipper (2001) claimed that related targets can reinstate the inhibitory processes that were applied to the prime.

Such retrieval theories can explain the present results (as well as those in Heil et al., 2004) by suggesting that semantic activation occurs and persists in the letter search task; however, any potential priming produced by the prime's residual activation is offset by the memory retrieval of the prime episode. According to Tipper (2001), this retrieval would reinstate the letter search process and inhibition for meaning that took place during the prime task. Alternatively, according to Neill and Mathis (1998), reinstating the letter search process alone could lead to interference with the appropriate target response (in this case, a pronunciation response). (See Hutchison, 2002, for discussion of the difficulty in discriminating between these two alternatives.)

\section{CONCLUSIONS}

The critical finding in the present study is that we obtained evidence of semantic activation in the letter search task while simultaneously observing the elimination of semantic priming. Searching for a letter in a word, while eliminating priming from that word, does not affect the initiation of semantic activation. Hence, any elimination of semantic priming from letter search cannot be taken as evidence against the claim that semantic activation is automatic. The results are consistent with models of activationbased suppression and target interference due to the inappropriate retrieval or reinstatement of prime processing.

\section{AUTHOR NOTE}

The authors thank Todd Kahan for helpful suggestions regarding the construction of materials for this project and Dave Balota, Jim Neely, and Tram Neill for their excellent comments on an earlier version of the manuscript. The authors also thank Eddie Furlong for his help in running the participants. Correspondence concerning this article should be addressed to K. A. Hutchison, Department of Psychology, Montana State University, 304 Traphagen Hall, Bozeman, MT 59717-3440 (e-mail: khutch@montana.edu).

\section{REFERENCES}

Algom, D., Chajut, E., \& Lev, S. (2004). A rational look at the emotional Stroop phenomenon: A generic slowdown, not a Stroop effect. Journal of Experimental Psychology: General, 133, 323-338.

Balota, D. A., Yap, M. J., Cortese, M. J., Hutchison, K. A., Kessler, B., Loftis, B., ET AL. (in press). The English Lexicon Project: A user's guide. Behavior Research Methods.

BESNER, D. (2001). The myth of ballistic processing: Evidence from Stroop's paradigm. Psychonomic Bulletin \& Review, 8, 324-330.

Besner, D., Smith, M. C., \& MacLeod, C. M. (1990). Visual word recognition: A dissociation of lexical and semantic processing. Journal of Experimental Psychology: Learning, Memory, \& Cognition, 16, 862-869.

Besner, D., Stolz, J. A., \& Boutilier, C. (1997). The Stroop effect and the myth of automaticity. Psychonomic Bulletin \& Review, 4, 221-225.

Besson, M., Fischler, I., Boaz, T., \& Raney, G. (1992). Effects of automatic associative activation on explicit and implicit memory tests. Journal of Experimental Psychology: Learning, Memory, \& Cognition, 18, 89-105.

Borowsky, R., \& Besner, D. (1993). Visual word recognition: A multistage activation model. Journal of Experimental Psychology: Learning, Memory, \& Cognition, 19, 813-840. 
Brown, M. S., Roberts, M. A., \& Besner, D. (2001). Semantic processing in visual word recognition: Activation blocking and domain specificity. Psychonomic Bulletin \& Review, 8, 778-784.

Catena, A., Fuentes, L. J., \& Tudela, P. (2002). Priming and interference effects can be dissociated in the Stroop task: New evidence in favor of the automaticity of word recognition. Psychonomic Bulletin \& Review, 9, 113-118

Chiappe, P. R., Smith, M. C., \& Besner, D. (1996). Semantic priming in visual word recognition: Activation blocking and domains of processing. Psychonomic Bulletin \& Review, 3, 249-253.

Deacon, D., Hewitt, S., Yang, C., \& Nagata, M. (2000). Eventrelated potential indices of semantic priming using masked and unmasked words: Evidence that the N400 does not reflect a post-lexical process. Cognitive Brain Research, 9, 137-146.

Friedrich, F. J., HeniK, A., \& Tzelgov, J. (1991). Automatic processes in lexical access and spreading activation. Journal of Experimental Psychology: Human Perception \& Performance, 17, 792-806.

GreEnBerg, J. H. (1966). Language universals, with special reference to feature hierarchies. The Hague: Mouton.

GREenBERG, J. H. (1987). The present status of markedness theory: A reply to Scheffler. Journal of Anthropological Research, 43, 367-374.

HASHER, L., \& ZACKs, R. T. (1979). Automatic and effortful processes in memory. Journal of Experimental Psychology: General, 108, 356-388.

Heil, M., Rolke, B., \& Pecchinenda, A. (2004). Automatic semantic activation is no myth: Semantic context effects on the N400 in the letter-search task in the absence of response time effects. Psychological Science, 15, 852-857.

Henik, A., Friedrich, F. J., \& KellogG, W. A. (1983). The dependence of semantic relatedness effects upon prime processing. Memory \& Cognition, 11, 366-373.

Henik, A., Friedrich, F. J., Tzelgov, J., \& Tramer, S. (1994). Capacity demands of automatic processes in semantic priming. Memory \& Cognition, 22, 157-168.

Houghton, G., \& Tipper, S. P. (1994). A model of inhibitory mechanisms in selective attention. In D. Dagenbach \& T. H. Carr (Eds.), Inhibitory processes in attention, memory, and language (pp. 53-112). San Diego: Academic Press.

Hutchison, K. A. (2002). The effect of asymmetrical association on positive and negative semantic priming. Memory \& Cognition, 30 1263-1276.

Hutchison, K. A. (2003). Is semantic priming due to association strength or featural overlap? A microanalytic review. Psychonomic Bulletin \& Review, 10, 785-813.

Kahan, T. A., Sellinger, J. J., \& Broman-Fulks, J. J. (2006). Associative and phonological priming effects after letter search on the prime. American Journal of Psychology, 119, 239-254.

KaWAмото, A. H. (1993). Nonlinear dynamics in the resolution of lexical ambiguity: A parallel distributed processing account. Journal of Memory \& Language, 32, 474-516.

Keele, S. W., \& NeILL, W. T. (1978). Mechanisms of attention. In E. C. Carterette \& M. P. Friedman (Eds.), Handbook of perception (Vol. 9, pp. 3-47). New York: Academic Press.

Kiefer, M., \& SpITZER, M. (2000). Time course of conscious and unconscious semantic brain activations. NeuroReport, 11, 2401-2407.

MACLEOD, C. M. (1991). Half a century of research on the Stroop effect: An integrative review. Psychological Bulletin, 109, 163-203.

MACNEvin, C., \& Besner, D. (2002). When are morphemic and semantic priming observed in visual word recognition? Canadian Journal of Experimental Psychology, 56, 112-119.

Malley, G. B., \& Strayer, D. L. (1995). Effect of stimulus repetition on positive and negative identity priming. Perception \& Psychophysics, 57, 657-667.

Manwell, L. A., Roberts, M. A., \& Besner, D. (2004). Single letter coloring and spatial cuing eliminates a semantic contribution to the Stroop effect. Psychonomic Bulletin \& Review, 11, 458-462.

Marí-Beffa, P., Fuentes, L. J., Catena, A., \& Houghton, G. (2000). Semantic priming in the prime task effect: Evidence of automatic semantic processing of distractors. Memory \& Cognition, 28, 635-647.

Marí-Beffa, P., Houghton, G., Estévez, A. F., \& Fuentes, L. J. (2000). Word-based grouping affects the prime-task effect on semantic priming. Journal of Experimental Psychology: Human Perception \& Performance, 26, 469-479.
Marí-Beffa, P., Valdés, B., Cullen, D. J. D., Catena, A., \& Houghton, G. (2005). ERP analyses of task effects on semantic processing from words. Cognitive Brain Research, 23, 293-305.

Masson, M. E. J. (1995). A distributed memory model of semantic priming. Journal of Experimental Psychology: Learning, Memory, \& Cognition, 21, 3-23.

MaXfield, L. (1997). Attention and semantic priming: A review of prime task effects. Consciousness \& Cognition, 6, 204-218.

McClelland, J. L., \& Rumelhart, D. E. (1981). An interactive activation model of context effects in letter perception: I. An account of basic findings. Psychological Review, 88, 375-407.

Milliken, B., Joordens, S., Merikle, P. M., \& Seiffert, A. E. (1998). Selective attention: A reevaluation of the implications of negative priming. Psychological Review, 105, 203-229.

Moss, H. E., Hare, M. L., DAY, P., \& Tyler, L. K. (1994). A distributed memory model of the associative boost in semantic priming. Connection Science, 6, 413-427.

NeELY, J. H. (1977). Semantic priming and retrieval from lexical memory: Roles of inhibitionless spreading activation and limited-capacity attention. Journal of Experimental Psychology: General, 106, 226-254.

NeEly, J. H., \& Kahan, T. A. (2001). Is semantic activation automatic? A critical re-evaluation. In H. L. Roediger III, J. S. Nairne, I. Neath, \& A. M. Surprenant (Eds.), The nature of remembering: Essays in honor of Robert G. Crowder (pp. 69-93). Washington, DC: American Psychological Association.

NeILL, W. T. (1997). Episodic retrieval in negative priming and repetition priming. Journal of Experimental Psychology: Learning, Memory, \& Cognition, 23, 1291-1305.

NeILl, W. T., \& Mathis, K. M. (1998). Transfer-inappropriate processing: Negative priming and related phenomena. In D. L. Medin (Ed.), The psychology of learning and motivation: Advances in research and theory (Vol. 38, pp. 1-44). San Diego: Academic Press.

Neill, W. T., Valdes, L. A., Terry, K. M., \& Gorfein, D. S. (1992). Persistence of negative priming: II. Evidence for episodic trace retrieval. Journal of Experimental Psychology: Learning, Memory, \& Cognition, 18, 993-1000.

Neill, W. T., \& Westberry, R. L. (1987). Selective attention and the suppression of cognitive noise. Journal of Experimental Psychology: Learning, Memory, \& Cognition, 13, 327-334.

Nelson, D. L., McEvoy, C. L., \& Schreiber, T. A. (1999). The University of South Florida word association, rhyme and word fragment norms. Available at w3.usf.edu/FreeAssociation.

Osterhout, L., \& Holcomb, P. J. (1995). Event-related potentials and language comprehension. In M. D. Rugg \& M. G. H. Coles (Eds.), Electrophysiology of mind: Event-related brain potentials and cognition (pp. 171-215). Oxford: Oxford University Press.

Plaut, D. C. (1995). Double dissociation without modularity: Evidence from connectionist neuropsychology. Journal of Clinical \& Experimental Neuropsychology, 17, 291-321.

Posner, M. I., \& SNyder, C. R. R. (1975). Attention and cognitive control. In R. L. Solso (Ed.), Information processing and cognition (pp. 55-85). Hillsdale, NJ: Erlbaum.

ReICHER, G. M. (1969). Perceptual recognition as a function of meaningfulness of stimulus material. Journal of Experimental Psychology, 81, 275-280.

SHIFFrin, R. M., \& SCHNEIDER, W. (1977). Controlled and automatic human information processing: II. Perceptual learning, automatic attending, and a general theory. Psychological Review, 84, 127-190.

Sмiтн, M. C. (1979). Contextual facilitation in a letter search task depends on how the prime is processed. Journal of Experimental Psychology: Human Perception \& Performance, 5, 239-251.

Smith, M. C., Bentin, S., \& SpaleK, T. M. (2001). Attention constraints of semantic activation during visual word recognition. Journal of Experimental Psychology: Learning, Memory, \& Cognition, 27, 1289-1298.

Smith, M. C., Theodor, L., \& Franklin, P. E. (1983). The relationship between contextual facilitation and depth of processing. Journal of Experimental Psychology: Learning, Memory, \& Cognition, 9, 697-712.

Stolz, J. A., \& Besner, D. (1996). Role of set in visual word recognition: Activation and activation blocking as nonautomatic processes. Journal of Experimental Psychology: Human Perception \& Performance, 22, 1166-1177.

Stolz, J. A., \& BeSNER, D. (1998). Levels of representation in visual 
word recognition: A dissociation between morphological and semantic processing. Journal of Experimental Psychology: Human Perception \& Performance, 24, 1642-1655.

Stolz, J. A., \& BeSNER, D. (1999). On the myth of automatic semantic activation in reading. Current Directions in Psychological Science, 8, 61-65.

StRoop, J. R. (1935). Studies of interference in serial verbal reactions. Journal of Experimental Psychology, 18, 643-662.

TIPPER, S. P. (1985). The negative priming effect: Inhibitory priming by ignored objects. Quarterly Journal of Experimental Psychology, 37A, 571-590.

TIPPER, S. P. (2001). Does negative priming reflect inhibitory mechanisms? A review and integration of conflicting views. Quarterly Journal of Experimental Psychology, 54A, 321-343.

Tipper, S. P., Weaver, B., \& Houghton, G. (1994). Behavioural goals determine inhibitory mechanisms of selective attention. Quarterly Journal of Experimental Psychology, 47A, 809-840.

VAn SElst, M., \& Joliceur, P. (1994). A solution to the effect of sample size on outlier elimination. Quarterly Journal of Experimental Psychology, 47A, 631-650.

Ziegler, J. C., Van Orden, G. C., \& Jacobs, A. M. (1997). Phonology can help or hurt the perception of print. Journal of Experimental Psychology: Human Perception \& Performance, 23, 845-860.

\section{NOTES}

1. An additional 2 (bias) $\times 2$ (letter presence) ANOVA was run only on the data from the 15 participants for whom we also were able to record accuracy. The pattern from this analysis was similar, with a marginally significant two-way interaction $\left[F(1,17)=5.56, M S_{\mathrm{e}}=5,715\right]$, reflecting the fact that the significant bias effect in the letter-present condition was marginally greater than the nonsignificant bias effect in the letterabsent condition.

2. We conducted an additional experiment to test the search difficulty hypothesis by replacing the letter search task with a vowel-counting task in which the meaning of the words is irrelevant to the required response. The positive and negative words had the same average number of vowels in roughly the same locations $(M=$ position 3.8 for positive words and 3.5 for negative words; $t<1$ for the difference in probe letter position between positive and negative words). The mean RTs based on 35 participants were 1,466 and $1,458 \mathrm{msec}$ for the positive and the negative bias words, respectively $(t<1)$.

\section{APPENDIX}

Bias Words and Letters (Positions)

Searched in Experiments 1 and 2

\begin{tabular}{|c|c|c|c|c|}
\hline \multirow[b]{2}{*}{ Word } & \multicolumn{3}{|c|}{ Experiment 1} & \multirow{2}{*}{$\begin{array}{c}\text { Experiment } 2 \\
\text { Letter }\end{array}$} \\
\hline & Early & Middle & Late & \\
\hline \multicolumn{5}{|c|}{ Positive Bias } \\
\hline Contain & $o(2)$ & $t(4)$ & $\mathrm{i}(6)$ & $c(1)$ \\
\hline Identical & d (2) & $\mathrm{t}(5)$ & a (8) & d (2) \\
\hline Match & $\mathrm{a}(2)$ & $\mathrm{t}(3)$ & c (4) & $t(3)$ \\
\hline Present & $r(2)$ & s (4) & n (6) & s (4) \\
\hline Same & a (2) & m(3) & e (4) & e (4) \\
\hline Yes & e (2) & e (2) & s (3) & y (1) \\
\hline \multicolumn{5}{|c|}{ Negative Bias } \\
\hline Absent & $\mathrm{b}(2)$ & s (3) & $\mathrm{n}(5)$ & $\mathrm{b}(2)$ \\
\hline Different & $\mathrm{i}(2)$ & r (6) & n (8) & $\mathrm{f}(3$ and 4$)$ \\
\hline Lacking & a (2) & $\mathrm{k}(4)$ & n (6) & $\mathrm{i}(5)$ \\
\hline Mismatch $^{\mathrm{a}}$ & i (2) & a (5) & c (7) & \\
\hline Missing & $\mathrm{i}(2$ and 5$)$ & s (3 and 4) & $\mathrm{n}(6)$ & m (1) \\
\hline No & $\mathrm{n}(1)$ & o (2) & o (2) & o (2) \\
\hline Omitted ${ }^{b}$ & & & & d (7) \\
\hline
\end{tabular}

(Manuscript received June 20, 2005

revision accepted for publication February 6, 2006.) 